METZLER LEXIKON ANTIKER AUTOREN 


\section{METZLER LEXIKON ANTIKER AUTOREN}

Mit 61 Abbildungen

Herausgegeben von

Oliver Schütze

Verlag J. B. Metzler

Stuttgart - Weimar 


\title{
Inhaltsverzeichnis
}

\author{
Vorwort $\mathrm{V}$ \\ Artikel A-Z I \\ Bibliographische Abkürzungen $77 \mathrm{I}$ \\ Glossar der Fachbegriffe 773 \\ Verzeichnis der Mitarbeiterinnen und Mitarbeiter 774 \\ Namenregister 779 \\ Bildquellen 79I
}

Die Deutsche Bibliothek - CIP-Einheitsaufnahme

Metzler-Lexikon antiker Autoren / hrsg. von Oliver Schütze.

- I. Aufl. - Stuttgart ; Weimar : Metzler, I997

ISBN 978-3-476-01547-1

ISBN 978-3-476-01547-1

ISBN 978-3-476-05282-7 (eBook)

DOI 10.1007/978-3-476-05282-7

Dieses Werk einschließlich aller seiner Teile ist urheberrechtlich geschützt. Jede Verwertung außerhalb der engen Grenzen des Urheberrechtsgesetzes ist ohne Zustimmung des Verlages unzulässig und strafbar. Das gilt insbesondere für Vervielfältigungen, Übersetzungen, Mikroverfilmungen und die Einspeicherung und Verarbeitung in elektronischen Systemen.

(C) 1997 Springer-Verlag GmbH Deutschland Ursprünglich erschienen bei J. B. Metzlersche Verlagsbuchhandlung und Carl Ernst Poeschel Verlag GmbH in Stuttgart 1997 


\section{Vorwort}

Das Metzler Lexikon antiker Autoren (MLAA) ist ein Nachschlagewerk für Leserinnen und Leser antiker Literatur und solche, die es werden wollen. Gerade für letztere gilt es, Schwellen zu überwinden, die durch eine von Textstellennachweisen und Testimonien beschwerte lexikalische Information nicht niedriger werden. Nun ist die Frage, wie antike Texte überliefert worden sind, von den Texten selbst nicht zu trennen; Philologen verfolgen detektivisch Überlieferungsspuren, fügen Textreste in Archäologenarbeit zusammen und befreien mit Hebammenkunst ganze Schriften aus anderen Textcorpora - diese besonderen Bedingungen sollte der Leser nicht vergessen, wenn er eine moderne Textausgabe in der Hand hält. Die im MLAA vorgelegten Artikel aber lassen diese Fragen zum größten Teil beiseite und versuchen statt dessen, die Informationen zu Leben, Werk und Wirkung in ein - kürzeres oder längeres - Porträt des jeweiligen Autors zu integrieren, das die Voraussetzungen und Eigenarten seines Schaffens erläutert und so eine Brücke baut zu unserem heutigen Literatur- und Kulturverständnis. Nicht selten wird hier gewertet und empfohlen die Verfasser, allesamt in der Philologie zu Hause, haben die Gelegenheit genutzt, einmal etwas freier über ihre 'Lieblingsautoren` zu schreiben.

Die Zielsetzung dieses Autorenlexikons bestimmt zugleich auch seine Grenzen: Weder kann es die in einer Literaturgeschichte mögliche Beschreibung von historischen Prozessen, etwa der Gattungsgeschichte oder bestimmter literarischer Strömungen, leisten noch sind hier alle oder auch nur die meisten der Autoren der Antike repräsentiert. Die 460 Artikel bieten aber den Großteil der Autoren, über die sich mehr Mitteilung machen läßt als lediglich die Konstatierung spärlicher Fragmente und Nachrichten, Autoren, deren Werk in der einen oder anderen Form Wirkung hatte bis in unsere Tage, sei es als dauerhafter Teil der Weltliteratur, monumentum aere perennius, wie es Horaz errichtet hat, sei es als Quellenwerk, das für die heutige historische Forschung von großem Wert ist - unter diesem Aspekt ist beispielsweise eine größere Anzahl von nur fragmentarisch überlieferten Historikern aufgenommen worden. Auch wurden die bedeutenden anonymen Texte und Sammlungen berücksichtigt, etwa der Alexander- und der Trojaroman, die großen Gedichtanthologien, die Annales, die Panegyriker, die gnostischen, hermetischen, orphischen und pseudopythagoreischen Schriften bis hin zu asklepischen Inschriften und Zauberpapyri. - Auch historische Entwicklungen und literarische Traditionsbildung lassen sich, geht man den impliziten Verweisen (auf einen 'Wald von Verweispfeilen wurde verzichtet) auf andere Artikel nach, durchaus verfolgen. Frappierend ist die Weltläufigkeit der antiken Literatur (erstaunlich allein schon, wie oft und wie weit gereist wurde!), die sich in der beständigen Bezugnahme auf Früheres und Gleichzeitiges ausdrückt - "Intertextualität«, und zwar über die ständig präsenten Homer und Platon, Vergil und Cicero hinaus, von der sich entfaltenden Tradition christlicher Literatur ganz zu schweigen.

Gegenstand des Lexikons ist die Literatur der griechisch-römischen MittelmeerOikumene von den Anfängen der überlieferten Literatur im 8. Jahrhundert v. Chr. bis zum Ausgang der Spätantike (mit einigen >Ausreißern in die byzantinische Zeit). 
Neben der klassischen "belletristischen" Tradition sind die Fachschriftsteller gebührend berücksichtigt; das kodifizierte Römische Recht Justinians oder Vitruvs Architekturtheorie haben die Moderne nicht weniger geprägt als die großen Epen. Das MLAA bringt u.a. Agrarschriftsteller, Astronomen, Enzyklopädisten, Geographen, Grammatiker, Juristen, Mathematiker, Mediziner, Militärschriftsteller, $\mathrm{Mu}$ siktheoretiker. - Für die Philosophen wurde auf die bewährten Texte aus dem Metzler Philosophen Lexikon (hrsg. von Bernd Lutz, 2., aktualisierte Auflage 1995) zurückgegriffen; sie sind hier, mit Ergänzungen zu den Literaturangaben, wiederabgedruckt. Viele Artikel zu weiteren Philosophen sind neu hinzugekommen, u.a. zu den Neuplatonikern und Aristoteleskommentatoren sowie zur sog. Zweiten Sophistik. - In welchem Maß Frauen in der Antike Literatur produziert haben, läßt sich kaum beurteilen; das wenige Überlieferte steht jedenfalls in deutlichem Gegensatz zu der häufigen Nennung von Dichterinnen und Philosophinnen zumindest in der griechischen Frühzeit. Alle Autorinnen, von denen aussagefähige Texte bzw. Fragmente oder wenigstens Zeugnisse vorhanden sind, werden im Lexikon vorgestellt: Anyte, Cornelia, Egeria, Erinna, Hypatia, Korinna, Moiro, Nossis, Perpetua, Praxilla, Proba, Sappho, Sulpicia.

Die Trennung der klassischen antiken Tradition von der christlichen, die aus pragmatischen Gründen und aufgrund verschiedener Forschungstraditionen meist vollzogen wird, ist im MLAA vermieden worden. Sehr schnell wird sich dem Leser der Artikel über die Kirchenväter, die Mönche, die Wanderprediger, die Häretiker, die Märtyrer und die Kirchenhistoriker offenbaren, wie eng die Verbindungen zwischen der klassischen Tradition und der um Identitätsfindung und Konsolidierung bemühten Christenheit waren. Vielfach versuchten die christlichen Autoren (z.B. Prudentius oder Laktanz) mit dem Rekurs auf das klassische Erbe die gebildeten, traditionsbewußten heidnischen Römer zu gewinnen; im Neuplatonismus gab es ohnehin fließende Übergänge von der heidnischen in die christliche Religion - oder aber den Versuch, eine konkurrenzfähige, philosophisch untermauerte pagane Religiosität dem attraktiven Christentum gegenüberzustellen. Der Kreis um den römischen Stadtpräfekten und heftigen Traditionalisten Symmachus, aus dem heraus spät noch einmal klassische Dichtung (Naucellius, Namatian) und die Pflege der klassischen Autoren (Servius, Macrobius) betrieben wurde, ist ohne die Gegnerschaft zu den Christen - Symmachus verlor ja den Streit um die Wiederaufstellung der Victoria-Statue im römischen Senat gegen den Bischof Ambrosius - kaum zu verstehen. Das MLAA ermöglicht den Blick auf zahlreiche Spielarten des Christenund des paganen Lebens in der spätantiken Welt und ihren literarischen Ausdruck.

Technisches: Die Literaturangaben der Artikel sind unterteilt in die Abschnitte "Editionen" und "Literatur". Unter "Ed." finden sich die wichtigsten Texteditionen, aber auch - nicht eigens von den kritischen Editionen unterschieden - zweisprachige Ausgaben mit einem bloßen Lesetext des Originals sowie einsprachige Übersetzungen, schließlich Kommentare. Bei den Übersetzungen wurden in der Regel die zweisprachigen Ausgaben der Reihe Tusculum (Zürich, München bzw. Düsseldorf), der Bibliothek der Alten Welt (Zürich, Stuttgart), der Loeb Classical Library (Cambridge, Mass. und London) und der Collection Budé (Paris) angefuihrt. Der 
Erscheinungsort gilt bei den Angaben zugleich als Hinweis, in welche Sprache übersetzt wurde. - Unter "Lit." ist grundlegende und aktuelle Einführungs- und Forschungsliteratur in Auswahl verzeichnet.

Griechische Begriffe erscheinen im Lexikon generell in Umschrift, wobei (langes) Eta und Omega mit Strich über dem Vokal bezeichnet sind und der Akzent, stellvertretend für alle Formen griechischer Akzente, lediglich einen Hinweis auf die Betonung des Wortes geben soll.

Am Schluß muß der Dank stehen: Er gilt vor allem den I 50 Autorinnen und Autoren, Fachwissenschaftlern von Graz bis Rostock, von Bern bis Dresden, die das Anliegen dieses Lexikons bereitwillig und rasch aufgegriffen und mit Anregungen und Empfehlungen nicht gespart haben. Ist das, in Zeiten von Sparbeschlüssen und Fachbereichsschließungen, als Zeichen zunehmender Lebendigkeit der Altertumswissenschaften zu nehmen? - Julia Schülli ist zu danken, die im Verlag die Redaktionsarbeit unterstützt und die Bildredaktion innegehabt hat. Stellvertretend für viele, die hier genannt werden sollten, danke ich Professor Kai Brodersen, dem das Lexikon eine ganze Reihe ausgezeichneter Verfasser verdankt und der in kritischer Sekunde mit Rat und Tat, nämlich eigenen Texten, zur Stelle war. Dr. Peter Habermehl danke ich ganz besonders: Er hat - ganz abgesehen von seinen eigenen Textbeiträgen - nicht nur treffsichere Empfehlungen für viele Artikelverfasser ausgesprochen, sondern auch die Artikelliste mahnend und ergänzend mitgestaltet. - Alle Lücken und Unzulänglichkeiten des Lexikons hat der Herausgeber allein zu verantworten. 